\title{
A 3D model for mechanistic control of drug release
}

\author{
J.A. Ferreira $^{\circ *}$ M. Grassi $\diamond^{\circ}$ E. Gudiño ${ }^{\circ}$ P. de Oliveira ${ }^{\circ} \S$ \\ ${ }^{\circ}$ CMUC, Department of Mathematics, University of Coimbra \\ 3001-454 Coimbra, Portugal \\ $\diamond$ Department of Engineering and Architecture, Trieste University \\ via Alfonso Valerio 6/A, Trieste, I-34127, Italy
}

\begin{abstract}
A 3D mathematical model for sorption/desorption by a cylindrical polymeric matrix with dispersed drug is proposed. The model is based on a system of partial differential equations coupled with boundary conditions over a moving boundary. We assume that the penetrant diffuses into a swelling matrix and causes a deformation which induces a stress driven diffusion and consequently a non-Fickian mass flux. A physically sound non linear dependence between strain and penetrant concentration is considered and introduced in a Boltzmann integral with a kernel computed from a Maxwell-Wiechert model. Numerical simulations show how the mechanistic behavior can have a role in drug delivery design.
\end{abstract}

\section{Introduction}

In this paper we study a 3D model of diffusion of a solvent into a cylindrical polymeric matrix containing drug and followed by the drug release. To describe drug release from a polymeric matrix, several models have been proposed [11, 12, 13, 15, 18, 25, 27]. However to the best of our knowledge, the influence of the mechanical properties of a swelling polymer in the sorption of a solvent and in the desorption of drug has not yet been considered in the literature. We propose a model where we combine non-Fickian sorption of the liquid agent, non-Fickian desorption coupled with non-linear dissolution and polymer swelling.

It is well known that the diffusion of a liquid agent into a polymeric sample cannot be completely described by Fick's classical law. The liquid strains the polymeric matrix that while swelling exerts a stress that acts as a barrier to the incoming fluid. To explain these phenomena several authors $[2,4,5,11,22,23,24]$ agree that a modified flux must be considered, that is

$$
\frac{\partial C_{l}}{\partial t}=-\operatorname{div}\left(J_{F}\left(C_{l}\right)+J_{N F}\left(\sigma_{l}\right)\right)
$$

where $C_{l}$ stands for the concentration of the penetrant, $J_{F}\left(C_{l}\right)=-\left(D\left(C_{l}\right) \nabla C_{l}\right)$ represents the Fickian part of the flux, $J_{N F}\left(\sigma_{l}\right)=-\left(D_{v}\left(C_{l}\right) \nabla \sigma_{l}\right)$ represents the non-Fickian part of the flux and $\sigma_{l}$ stands for the stress. The functions $D\left(C_{l}\right)$ and $D_{v}\left(C_{l}\right)$ represent respectively the Fickian

\footnotetext{
*ferreira@mat.uc.pt

${ }^{\dagger}$ mariog@dicamp.univ.trieste.it

${ }^{\ddagger}$ egudino@gmail.com

${ }^{\S}$ poliveir@mat.uc.pt
} 
diffusion coefficient and a viscoelastic diffusion coefficient. Since equation (1) is coupled with a stress evolution equation, the strain $\epsilon$ is introduced as a third variable. Many different constitutive relationships between stress and strain have been considered in the literature $[2,4,3,5,16,17]$. In this paper we propose the use of a Boltzmann integral of type

$$
\sigma(t)=-\int_{0}^{t} E(t-s) \frac{\partial \epsilon}{\partial s}(s) d s
$$

where $E(t)$ is the relaxation modulus corresponding to a Maxwell-Wiechert model [1, 19]. We note that the minus sign in (2) means that as the penetrant solvent strains the polymeric matrix, a stress of opposite sign is developed. When (2) is introduced in (1), the strain must be eliminated as a variable. Therefore we consider a non-linear functional relation between strain and concentration

$$
\epsilon=f\left(C_{l}\right)
$$

where $f$ is established using physical arguments [7], as we will briefly describe in Section 2 .

We assume that as the solvent penetrates the polymer the drug is present in two states (dissolved and undissolved) and also that the drug release is controlled by both non-Fickian diffusion and a non-linear dissolution. As the amount of dissolved drug does not induce, locally, any kind of re-arrangement of the polymeric chains, the non-Fickian character of the diffusion equation that describes the drug release is due to solvent uptake.

We track the moving front resulting from the swelling of the polymer by considering a volume conservation equation [25]. As we assume the swelling to be independent in the radial and axial directions we use this volume conservation equation to track separately both of the moving fronts [9].

In Section 2 we establish a mathematical model to describe the absorbtion and drug release. In Section 3 we introduce a volume conservation equation to describe the swelling of the polymeric matrix. In Section 4 an Implicit-Explicit (IMEX) numerical scheme is used to numerically solve the model. In Section 5 some plots are presented to illustrate the behavior of the numerical solutions. Finally in Section 6 some conclusions are addressed.

\section{Mathematical model}

Let us consider a cylindrical polymeric matrix, with initial solid drug loading $C_{s}^{0}$. As the solvent penetrates the polymeric matrix, solid drug dissolves and dissolved drug diffuses out. The following assumptions are made in the model: (a) swelling is homogeneous and independent in the radial and axial directions; (b) the transport of liquid within the polymer occurs by non-Fickian diffusion; (c) the transport of drug out of the polymer occurs by non-Fickian diffusion associated with solvent uptake and non-linear dissolution; (d) the positions of the polymer swelling front and dissolution front coincide; and (e) a perfect sink condition is maintained for the drug and equilibrium concentrations are maintained for the liquid.

To describe the viscoelastic behavior of the polymer, we consider a generalized Maxwell-Wiechert model $[1,19]$ with $m+1$ arms in parallel as shown in figure 1 . Consequently the relaxation modulus $E(t)$ is represented by

$$
E(t)=\sum_{k=1}^{m} E_{k} e^{\frac{-t}{\tau_{k}}}+E_{0}
$$


where the $E_{k}^{\prime} s$ are the Young modulus of the spring elements, the $\mu_{k}^{\prime} s$ represent the viscosity, $\tau_{k}=\frac{\mu_{k}}{E_{k}}$ are the relaxation times associated to each of the $m$ Maxwell fluid arms and $E_{0}$ stands for the Young modulus of the free spring.

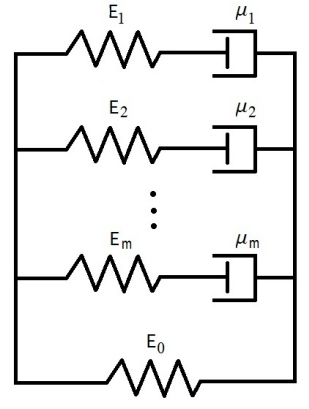

Figure 1: Generalized Maxwell-Wiechert model

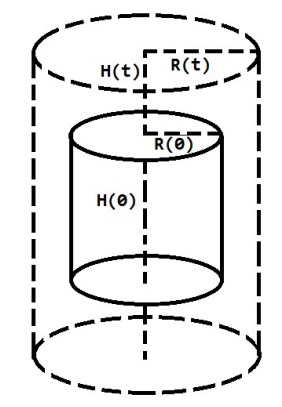

Figure 2: Cylindrical domain

Let $C_{l}$ denote the concentration of the liquid solvent. The functional relation between $C_{l}$ and the strain $\epsilon$, is defined by

$$
f_{l}\left(C_{l}\right)=\frac{C_{l}}{\rho_{l}-C_{l}} .
$$

where $\rho_{l}$ denotes the density of the liquid [7]. Then from (2), (4) and (5) we conclude that the stress associated to solvent uptake and exerted by the polymer is defined as

$$
\sigma_{l}=-\left(\sum_{k=0}^{m} E_{k}\right) \nabla f_{l}+\int_{0}^{t}\left(\sum_{k=1}^{m} \frac{E_{k}}{\tau_{k}} e^{-\frac{t-s}{\tau_{k}}}\right) \nabla f_{l}(s) d s .
$$

The evolution of solvent penetration, drug diffusion and dissolution are described by the following equations on the domain $\Omega \subset \mathbb{R}^{3}$ and for $t>0$,

$$
\begin{aligned}
\frac{\partial C_{l}}{\partial t} & =\nabla \cdot\left(-J_{F}\left(C_{l}\right)-J_{N F}\left(\sigma_{l}\right)\right)=\nabla \cdot\left(D_{l}\left(C_{l}\right) \nabla C_{l}+D_{v}\left(C_{l}\right) \nabla \sigma_{l}\right) \\
\frac{\partial C_{d}}{\partial t} & =\nabla \cdot\left(D_{d}\left(C_{l}\right) \nabla C_{d}+v\left(C_{l}\right) C_{d}\right)+K_{d}\left(\frac{C_{s}-C_{d}}{C_{s}}\right) C_{l} \\
\frac{\partial C_{s}}{\partial t} & =-K_{d}\left(\frac{C_{s}-C_{d}}{C_{s}}\right) C_{l}
\end{aligned}
$$

where $C_{d}, C_{s}$ denote the concentration of dissolved and solid drug respectively, $D_{l}, D_{d}$ the diffusion coefficients of the liquid solvent and the dissolved drug respectively, $K_{d}$ denotes the constant dissolution rate of the drug and $v$ is defined as

$$
v\left(C_{l}\right)=D_{v}\left(C_{l}\right) \frac{\nabla \sigma_{l}}{C_{l}}
$$

Equation (7) states that liquid solvent transport is due to Fickian $\left(J_{F}\right)$ and non-Fickian $\left(J_{N F}\right)$ diffusion. At the same time, equation (8) states that the local concentration of dissolved drug depends on Fickian diffusion $\left(D_{d}\left(C_{l}\right) \nabla C_{d}\right)$, convection $\left(v\left(C_{l}\right)\right)$ and on solid drug dissolution. While 
solid drug dissolution can take place provided that $C_{l}>0$, the velocity field $v$ is due to the stress induced by the solvent income. Indeed, we have that $v=J_{N F} / C_{l}$.

To establish a functional relation for the viscoelastic diffusion coefficient $D_{v}$ we follow [7]. We begin by assuming the existence of a stress gradient $\nabla \sigma_{l}$, that implies the existence of a velocity field $\nu$. Then the non-Fickian flux $J_{N F}$ can be interpreted as a convective field of form

$$
J_{N F}=\nu C_{l} \text {. }
$$

If we compute the velocity field $\nu$ using the Hagen-Poiseuille equation, we have

$$
\nu=-\frac{R^{2}}{8 \hat{\mu}} \nabla p
$$

where $R$ stands for the radius of a virtual cross section of the polymeric sample available for the convective flux, $p$ is the pressure drop and $\hat{\mu}$ represents the viscosity of a polymer-solvent solution characterized by a liquid (or solvent) concentration equal to $C_{l}$ (local solvent concentration). Thus from (10) and (11) and identifying the pressure $p$ with the viscoelastic stress $\sigma_{l}$, we conclude that

$$
D_{v}\left(C_{l}\right)=\frac{R^{2} C_{l}}{8 \hat{\mu}}
$$

Let $m_{l}$ and $V_{l}$ represent the mass and volume of the solvent respectively. If $\rho_{l}$ represents its density, then $m_{l}=\rho_{l} V_{l}$ and $C_{l}=\frac{m_{l}}{V_{0}+V_{l}}$, where $V_{0}$ is the volume of the polymeric matrix in the dry state. We conclude then that

$$
V_{l}=\frac{C_{l}}{\rho_{l}-C_{l}} V_{0}
$$

and as $V_{0}=\Delta x_{0} S$, we have

$$
\frac{V_{l}}{\Delta x_{0}}=\frac{C_{l}}{\rho_{l}-C_{l}} S .
$$

The first member in (13) can be interpreted as a virtual cross section $S_{v}$ available for convective flow. As $S_{v}=\pi R^{2}$ and $S=\pi R_{0}^{2}$ where $R_{0}$ is the radius of the dry sample, we deduce the form of the viscoelastic coefficient $D_{v}$,

$$
D_{v}\left(C_{l}\right)=\frac{R_{0}^{2} C_{l}^{2}}{8 \hat{\mu}\left(\rho_{l}-C_{l}\right)} .
$$

A Fujita-type [10] exponential dependence for $D_{l}\left(C_{l}\right)$ and $D_{d}\left(C_{l}\right)$ is assumed with

$$
\begin{aligned}
& D_{l}\left(C_{l}\right)=D_{e q_{l}} \exp \left(-\beta_{l}\left(1-\frac{C_{l}}{C_{l}^{e}}\right)\right), \\
& D_{d}\left(C_{l}\right)=D_{e q_{d}} \exp \left(-\beta_{d}\left(1-\frac{C_{l}}{C_{l}^{e}}\right)\right),
\end{aligned}
$$

where $D_{e q_{l}}, D_{e q_{d}}$ denote respectively the diffusion coefficients of the liquid solvent and the dissolved drug in the fully swollen sample and $\beta_{l}, \beta_{d}$ dimensionless positive constants.

We consider a cylindrical domain $\Omega \subset \mathbb{R}^{3}$ with initial radius $R_{0}$ and height $H_{0}$ (Figure 2 ). Due to the symmetry in $\theta$ direction, the three dimensional problem is reduced to a two dimensional 
case. Therefore equations (7)-(9) can be rewritten in cylindrical coordinates as

$$
\begin{aligned}
\frac{\partial C_{l}}{\partial t}= & \frac{1}{r} \frac{\partial}{\partial r}\left(r D_{l}\left(C_{l}\right) \frac{\partial C_{l}}{\partial r}+r D_{v}\left(C_{l}\right) \frac{\partial \sigma_{l}}{\partial r}\right)+\frac{\partial}{\partial z}\left(D_{l}\left(C_{l}\right) \frac{\partial C_{l}}{\partial z}+D_{v}\left(C_{l}\right) \frac{\partial \sigma_{l}}{\partial z}\right), \\
\frac{\partial C_{d}}{\partial t}= & \frac{1}{r} \frac{\partial}{\partial r}\left(r D_{d}\left(C_{l}\right) \frac{\partial C_{d}}{\partial r}+r v\left(C_{l}\right) C_{d}\right)+\frac{\partial}{\partial z}\left(D_{d}\left(C_{l}\right) \frac{\partial C_{d}}{\partial z}+v\left(C_{l}\right) C_{d}\right) \\
& +K_{d}\left(\frac{C_{s}-C_{d}}{C_{s}}\right) C_{l}, \\
\frac{\partial C_{s}}{\partial t}= & -K_{d}\left(\frac{C_{s}-C_{d}}{C_{s}}\right) C_{l},
\end{aligned}
$$

where $0<r<R(t), 0<z<H(t)$ and $t>0$. Equations (17)-(19) are completed with initial conditions

$$
C_{l}=C_{l}^{0}, C_{d}=0, C_{s}=C_{s}^{0}: \text { for } t=0,0 \leq r \leq R_{0}, 0 \leq z \leq H_{0},
$$

where $C_{l}^{0}, C_{s}^{0} \in \mathbb{R}$ are positive constants. At the cylinder surface the boundary conditions are

$$
C_{l}=C_{l}^{e}, C_{d}=0: \text { for } t>0, r=R(t), 0 \leq z \leq H(t) \text { and } z=H(t), 0 \leq r \leq R(t),
$$

where $C_{l}^{e} \in \mathbb{R}$ is a positive constant representing the concentration of the liquid agent in the exterior of the cylinder. Symmetry conditions are applied at the center of the matrix, hence we also have that

$$
\begin{array}{ll}
\frac{\partial C_{l}}{\partial z}=\frac{\partial C_{d}}{\partial z}=0 \quad: \quad \text { for } t>0, r=0,0 \leq z \leq H(t) \\
\frac{\partial C_{l}}{\partial r}=\frac{\partial C_{d}}{\partial r}=0 \quad: \quad \text { for } t>0, z=0,0 \leq r \leq R(t) .
\end{array}
$$

The model (17)-(22) is based on a new interpretation of the non-Fickian flux and the establishment of non-linear functional relations for the strain $\epsilon$ and the diffusion coefficient $D_{v}$. The rationale underlying the approach to non-Fickian diffusion used in this paper is described in [6] and [7]. Although many authors have dealt in the past with the description of drug diffusion in a (polymeric) matrix system [20], at our knowledge, our model is one of the most complete if we neglect possible re-crystallization effect upon dissolution [12]. In addition, the main advantage of our model is that it allows the incorporation of experimental rheological information about the polymer-solvent system. This makes the model appropriate for both data fitting and quantitative prediction.

\section{Tracking of the swelling fronts}

In order to track the moving fronts due to swelling, we consider the following conservation equation, where the total volume of the matrix is the sum of water, dissolved and undissolved drug volumes. We have

$$
\pi R^{2}(t) H(t)=\int_{0}^{H(t)} \int_{0}^{R(t)} 2 \pi r\left[\frac{1}{\rho_{l}} C_{l}(r, z, t)+\frac{1}{\rho_{d}}\left(C_{d}(r, z, t)+C_{s}(r, z, t)\right)\right] d r d z+\frac{m_{0}}{\rho_{p}},
$$


where $\rho_{p}$ and $\rho_{d}$ denote the density of the polymer and the drug respectively, $m_{0}$ represent the initial mass of the dry polymeric matrix.

Since we assume the swelling to be independent in the two directions, by taking time derivatives in (23), the moving fronts in the radial and axial direction can be separately tracked.

To track the moving front in the radial direction we begin by fixing $H(t)=H$ and taking time derivative in $(23)$ to obtain

$$
\begin{aligned}
R(t) H \frac{\partial R(t)}{\partial t}= & \int_{0}^{H} \int_{0}^{R(t)} r\left[\frac{1}{\rho_{l}} \frac{\partial C_{l}}{\partial t}(r, z, t)+\frac{1}{\rho_{d}} \frac{\partial}{\partial t}\left(C_{d}(r, z, t)+C_{s}(r, z, t)\right)\right] d r d z \\
& +\int_{0}^{H} R(t) \frac{\partial R(t)}{\partial t}\left(\frac{C_{l}^{e}}{\rho_{l}}+\frac{\left.C_{s}\right|_{R(t)}}{\rho_{d}}\right) d z .
\end{aligned}
$$

As we have that

$$
\begin{aligned}
& \int_{0}^{H} \int_{0}^{R(t)} \frac{r}{\rho_{l}} \frac{\partial C_{l}}{\partial t}(r, z, t) d r d z \\
= & \int_{0}^{H} \frac{R(t)}{\rho_{l}}\left(D_{l}\left(C_{l}(R(t), z, t)\right) \frac{\partial C_{l}}{\partial r}(R(t), z, t)+D_{v}\left(C_{l}(R(t), z, t)\right) \frac{\partial \sigma_{l}}{\partial r}(R(t), z, t)\right)
\end{aligned}
$$

and

$$
\int_{0}^{H} \int_{0}^{R(t)} r \frac{1}{\rho_{d}} \frac{\partial}{\partial t}\left(C_{d}(r, z, t)+C_{s}(r, z, t)\right) d r d z=\int_{0}^{H} \frac{R(t)}{\rho_{d}} D_{d}\left(C_{l}(R(t), z, t)\right) \frac{\partial C_{d}}{\partial r}(R(t), z, t) d z,(26
$$

it follows from (24)-(26) that

$$
\begin{aligned}
\left(1-\frac{C_{l}^{e}}{\rho_{l}}-\frac{\left.C_{s}\right|_{R(t)}}{\rho_{d}}\right) H \frac{\partial R(t)}{\partial t}= & \int_{0}^{H}\left[\frac{1}{\rho_{l}}\left(D_{l}\left(C_{l}^{e}\right) \frac{\partial C_{l}}{\partial r}(R(t), z, t)+D_{v}\left(C_{l}^{e}\right) \frac{\partial \sigma_{l}}{\partial r}(R(t), z, t)\right)\right. \\
& \left.+\frac{1}{\rho_{d}} D_{d}\left(C_{l}^{e}\right) \frac{\partial C_{d}}{\partial r}(R(t), z, t)\right] d z
\end{aligned}
$$

To track the moving front in the axial direction, we fix $R(t)=R$ and proceeding as before we deduce

$$
\begin{aligned}
\left(1-\frac{C_{l}^{e}}{\rho_{l}}-\frac{\left.C_{s}\right|_{H(t)}}{\rho_{d}}\right) R^{2} \frac{\partial H(t)}{\partial t}= & 2 \int_{0}^{R}\left[\frac{1}{\rho_{l}}\left(D_{l}\left(C_{l}^{e}\right) \frac{\partial C_{l}}{\partial z}(r, H(t), t)+D_{v}\left(C_{l}^{e}\right) \frac{\partial \sigma_{l}}{\partial z}(r, H(t), t)\right)\right. \\
& \left.+\frac{1}{\rho_{d}} D_{d}\left(C_{l}^{e}\right) \frac{\partial C_{d}}{\partial z}(r, H(t), t)\right] r d r .
\end{aligned}
$$

We note that if no mechanistic effects are taken into account and the drug is considered to exist only in the dissolved state then (27) and (28) reduce to the moving boundary conditions in [9].

\section{Numerical scheme}

In this section we propose a coupled Implicit-Explicit (IMEX) method to solve the initial-boundary value problem (17)-(22) and (27), (28).

In $[0, T]$ we consider a grid $P=\left\{t_{n}, n=0,1, \ldots, M\right\}$ with $t_{0}=0, t_{M}=T$ and $t_{n}-t_{n-1}=\Delta t$. We denote by $D_{-t}$ the usual backward finite difference operator with respect to the time variable. 
As the spatial boundary is changing in time, we consider in the initial interval $\left[0, R_{0}\right]$ a uniform grid $I\left(t_{0}\right)=\left\{r_{i}, i=0,1, . ., N\left(t_{0}\right)\right\}$ with $r_{0}=0, r_{N\left(t_{0}\right)}=R_{0}$ and $r_{i}-r_{i-1}=\Delta r$. Then in each interval $\left[0, R\left(t_{n}\right)\right]$ we consider a non-uniform grid $I\left(t_{n}\right)=\left\{r_{i}, i=0,1, . ., N\left(t_{n}\right)\right\}$ with $r_{0}=0$, $r_{N\left(t_{n}\right)}=R\left(t_{n}\right)$ and $r_{i}-r_{i-1}=\Delta r_{i}$. We denote by $D_{-r}$ and $D_{r}$ the usual backward and forward finite difference operator with respect to the space variable $r$.

Analogously in the initial interval $\left[0, H_{0}\right]$ we consider a uniform grid $J\left(t_{0}\right)=\left\{z_{j}, j=0,1, . ., N\left(t_{0}\right)\right\}$ with $z_{0}=0, z_{K\left(t_{0}\right)}=H_{0}$ and $z_{j}-z_{j-1}=\Delta z$. Then in each interval $\left[0, H\left(t_{n}\right)\right]$ we consider a nonuniform grid $J\left(t_{n}\right)=\left\{z_{j}, j=0,1, \ldots, K\left(t_{n}\right)\right\}$ with $z_{0}=0, z_{K\left(t_{n}\right)}=H\left(t_{n}\right)$ and $z_{j}-z_{j-1}=\Delta z_{j}$. We denote by $D_{-z}$ and $D_{z}$ the usual backward and forward finite difference operator with respect to the space variable $z$.

Let $M_{h_{r}}$ and $M_{h_{z}}$ be defined as

$$
\begin{aligned}
& M_{h_{r}} u_{h}\left(r_{i}, z_{j}\right)=\frac{1}{2}\left(u_{h}\left(r_{i-1}, z_{j}\right)+u_{h}\left(r_{i}, z_{j}\right)\right), \\
& M_{h_{z}} u_{h}\left(r_{i}, z_{j}\right)=\frac{1}{2}\left(u_{h}\left(r_{i}, z_{j-1}\right)+u_{h}\left(r_{i}, z_{j}\right)\right),
\end{aligned}
$$

then we introduce the following notations

$$
\begin{aligned}
I M_{l, r}\left(r_{i}, z_{j}, t_{n}\right) & =D_{l}\left(M_{h_{r}} C_{l_{h}}^{n-1}\left(r_{i}, z_{j}\right)\right) D_{-r} C_{l_{h}}^{n}\left(r_{i}, z_{j}\right), \\
I M_{l, z}\left(r_{i}, z_{j}, t_{n}\right) & =D_{l}\left(M_{h_{z}} C_{l_{h}}^{n-1}\left(r_{i}, z_{j}\right)\right) D_{-z} C_{l_{h}}^{n}\left(r_{i}, z_{j}\right), \\
E X_{l, r}\left(r_{i}, z_{j}, t_{n-1}\right) & =D_{v}\left(M_{h_{r}} C_{l_{h}}^{n-1}\left(r_{i}, z_{j}\right)\right) D_{-r} \sigma_{l_{h}}^{n-1}\left(r_{i}, z_{j}\right), \\
E X_{l, z}\left(r_{i}, z_{j}, t_{n-1}\right) & =D_{v}\left(M_{h_{z}} C_{l_{h}}^{n-1}\left(r_{i}, z_{j}\right)\right) D_{-z} \sigma_{l_{h}}^{n-1}\left(r_{i}, z_{j}\right) .
\end{aligned}
$$

and

$$
\begin{aligned}
I M_{d, r}\left(r_{i}, z_{j}, t_{n}\right) & =D_{d}\left(M_{h_{r}} C_{l_{h}}^{n}\left(r_{i}, z_{j}\right)\right) D_{-r} C_{d_{h}}^{n}\left(r_{i}, z_{j}\right), \\
I M_{d, z}\left(r_{i}, z_{j}, t_{n}\right) & =D_{d}\left(M_{h_{z}} C_{l_{h}}^{n}\left(r_{i}, z_{j}\right)\right) D_{-z} C_{d_{h}}^{n}\left(r_{i}, z_{j}\right), \\
E X_{d, r}\left(r_{i}, z_{j}, t_{n-1}\right) & =D_{v}\left(M_{h_{r}} C_{l_{h}}^{n}\left(r_{i}, z_{j}\right)\right) \frac{M_{h_{r}} C_{d_{h}}^{n}\left(r_{i}, z_{j}\right)}{M_{h_{r}} C_{l_{h}}^{n}\left(r_{i}, z_{j}\right)} D_{-r} \sigma_{l_{h}}\left(C_{l_{h}}^{n}\left(r_{i}, z_{j}\right)\right), \\
E X_{d, z}\left(r_{i}, z_{j}, t_{n-1}\right) & =D_{v}\left(M_{h_{z}} C_{l_{h}}^{n}\left(r_{i}, z_{j}\right)\right) \frac{M_{h_{z}} C_{d_{h}}^{n}\left(r_{i}, z_{j}\right)}{M_{h_{z}} C_{l_{h}}^{n}\left(r_{i}, z_{j}\right)} D_{-z} \sigma_{l_{h}}\left(C_{l_{h}}^{n}\left(r_{i}, z_{j}\right)\right),
\end{aligned}
$$

where we have used $I M$ and $E X$ to underline the implicit and the explicit character of the discretization respectively.

The IMEX method for (17)-(19) is defined by

$$
\begin{aligned}
D_{-t} C_{l_{h}}^{n}\left(r_{i}, z_{j}\right)= & \frac{1}{r_{i}} D_{r}\left(\left(M_{h_{r}} r_{i}\right) I M_{l, r}\left(r_{i}, z_{j}, t_{n}\right)+\left(M_{h_{r}} r_{i}\right) E X_{l, r}\left(r_{i}, z_{j}, t_{n-1}\right)\right) \\
& +D_{z}\left(I M_{l, z}\left(r_{i}, z_{j}, t_{n}\right)+E X_{l, z}\left(r_{i}, z_{j}, t_{n-1}\right)\right), \\
D_{-t} C_{d_{h}}^{n}\left(r_{i}, z_{j}\right)= & \frac{1}{r_{i}} D_{r}\left(\left(M_{h_{r}} r_{i}\right) I M_{d, r}\left(r_{i}, z_{j}, t_{n}\right)+\left(M_{h_{r}} r_{i}\right) E X_{d, r}\left(r_{i}, z_{j}, t_{n-1}\right)\right) \\
& +D_{z}\left(I M_{d, z}\left(r_{i}, z_{j}, t_{n}\right)+E X_{d, z}\left(r_{i}, z_{j}, t_{n-1}\right)\right) \\
& +K_{d}\left(\frac{C_{s_{h}}^{n-1}\left(r_{i}, z_{j}\right)-C_{d_{h}}^{n-1}\left(r_{i}, z_{j}\right)}{C_{s_{h}}^{n-1}\left(r_{i}, z_{j}\right)}\right) C_{l_{h}}^{n}\left(r_{i}, z_{j}\right), \\
D_{-t} C_{s_{h}}^{n}\left(r_{i}, z_{j}\right)= & -K_{d}\left(\frac{C_{s_{h}}^{n-1}\left(r_{i}, z_{j}\right)-C_{d_{h}}^{n}\left(r_{i}, z_{j}\right)}{C_{s_{h}}^{n-1}\left(r_{i}, z_{j}\right)}\right) C_{l_{h}}^{n}\left(r_{i}, z_{j}\right),
\end{aligned}
$$


with initial conditions

$$
C_{l_{h}}^{0}=C_{l}^{0}, C_{d_{h}}^{0}=0, C_{s_{h}}=C_{s}^{0}: \text { for } t=0,0 \leq r_{i} \leq R_{0}, 0 \leq z_{j} \leq H_{0},
$$

boundary conditions on the cylinder surface

$$
C_{l_{h}}=C_{l}^{e}, C_{d_{h}}=0 \text { : for } n>0, r_{i}=R\left(t_{n}\right), 0 \leq z_{j} \leq H\left(t_{n}\right) \text { and } z_{j}=H\left(t_{n}\right), 0 \leq r_{i} \leq R\left(t_{n}\right),
$$

and boundary conditions at the symmetric axes

$$
\begin{aligned}
& D_{-z} C_{l_{h}}=D_{-z} C_{d_{h}}=0 \quad: \quad \text { for } n>0, r_{i}=0,0 \leq z_{j} \leq H\left(t_{n}\right) \\
& D_{-r} C_{l_{h}}=D_{-r} C_{d_{h}}=0 \quad: \quad \text { for } n>0, z_{j}=0,0 \leq r_{i} \leq R\left(t_{n}\right)
\end{aligned}
$$

The moving front defined by (27) and (28) is tracked with the following equations

$$
\begin{aligned}
\left(1-\frac{C_{l}^{e}}{\rho_{l}}-\frac{\left.C_{s}\right|_{R\left(t_{n}\right)}}{\rho_{d}}\right) H\left(t_{n}\right) D_{-t} R\left(t_{n+1}\right)= & \Delta z \sum_{j=1}^{K\left(t_{n}\right)} \frac{1}{\rho_{l}}\left(D_{l}\left(C_{l}^{e}\right) D_{-r} C_{l_{h}}^{n}\left(R\left(t_{n}\right), z_{j}\right)\right. \\
& \left.+D_{v}\left(C_{l}^{e}\right) D_{-r} \sigma_{l_{h}}^{n}\left(R\left(t_{n}\right), z_{j}\right)\right) \\
& +\frac{1}{\rho_{d}} D_{d}\left(C_{l}^{e}\right) D_{-r} C_{d_{h}}^{n}\left(R\left(t_{n}\right), z_{j}\right.
\end{aligned}
$$

and

$$
\begin{aligned}
\left(1-\frac{C_{l}^{e}}{\rho_{l}}-\frac{\left.C_{s}\right|_{H\left(t_{n}\right)}}{\rho_{d}}\right) R^{2}\left(t_{n}\right) D_{-t} H\left(t_{n+1}\right)= & 2 \Delta r \sum_{i=1}^{N\left(t_{n}\right)} \frac{r_{i}}{\rho_{l}}\left(D_{l}\left(C_{l}^{e}\right) D_{-r} C_{l_{h}}^{n}\left(r_{i}, H\left(t_{n}\right)\right)\right. \\
& \left.+D_{v}\left(C_{l}^{e}\right) D_{-r} \sigma_{l_{h}}^{n}\left(r_{i}, H\left(t_{n}\right)\right)\right) \\
& +\frac{r_{i}}{\rho_{d}} D_{d}\left(C_{l}^{e}\right) D_{-r} C_{d_{h}}^{n}\left(r_{i}, H\left(t_{n}\right)\right) .
\end{aligned}
$$

We compute the concentration profiles at time step $t_{n}$ using the known concentration profiles at $t_{n-1}$ with boundary conditions (33) and (34). Then we use (35) and (36) to obtain the new front position for the next time step.

The stability and convergence of the method (29)-(36) when the boundary is fixed and no dispersed drug is present was introduced in [6] for the one-dimensional case. The authors showed that it is second order convergent in space and first order convergent in time. A new approach, that does not follow the usual splitting of the global error using the solution of an elliptic equation induced by the integro-differential equation, introduced by Wheeler in[26] and largely followed in the literature, was considered.

In the case of a free boundary problem the analysis of stability and convergence results for the method (29)-(36) is a very difficult problem, and cannot be studied with the approach followed in [6] nor with classical numerical analysis approaches. Nonetheless we exhibit in the next section some numerical results that show clear evidence of a physically sound behavior of the model. 


\section{$5 \quad$ Numerical Results}

In what follows we exhibit some numerical results for the initial-boundary value problem (17)-(22) and (27), (28) using the method (29)-(36). In (4) we consider $m=1$, that is a Maxwell fluid arm in parallel with a free spring. The following values for the parameters have been considered,

$$
\begin{aligned}
R_{0} & =1 \times 10^{-3} \mathrm{~m}, \Delta r_{\max }=5 \times 10^{-5} \mathrm{~m}, H_{0}=1 \times 10^{-3} \mathrm{~m}, \Delta z_{\max }=5 \times 10^{-5} \mathrm{~m}, \\
D_{e q_{l}} & =3.74 \times 10^{-9} \mathrm{~m}^{2} / \mathrm{s}, D_{e q_{d}}=2.72 \times 10^{-10} \mathrm{~m}^{2} / \mathrm{s}, \beta_{l}=0.8, \beta_{d}=0.5, \hat{\mu}=20 \times 10^{5} \mathrm{Pas}, \\
\rho_{l} & =1000 \mathrm{~kg} / \mathrm{m}^{3}, \rho_{p}=1175 \mathrm{~kg} / \mathrm{m}^{3}, \rho_{d}=1400 \mathrm{~kg} / \mathrm{m}^{3}, E_{1}=9 \times 10^{3} \mathrm{~Pa}, E_{0}=1 \times 10^{3} \mathrm{~Pa}, \\
\mu_{1} & =225 \times 10^{4} \mathrm{Pas}, C_{l}^{e}=755 \mathrm{Kg} / \mathrm{m}^{3}, C_{l}^{0}=0 \mathrm{Kg} / \mathrm{m}^{3}, C_{s}^{0}=4.5 \mathrm{Kg} / \mathrm{m}^{3}, \\
K_{d} & =1 \times 10^{-2} \mathrm{~s}^{-1} \text { and } \Delta t=0.01 \mathrm{~s} .
\end{aligned}
$$

In Figures 3 we plotted the behavior of the concentration of the liquid solvent as it diffuses into the polymeric cylinder at $t=1 \mathrm{~s}, t=8 \mathrm{~s}, t=15 \mathrm{~s}$ and $t=25 \mathrm{~s}$. A quarter of the cylinder cross section was modeled due to symmetries. The axes $z$ and $r$ correspond to the inner part of the cylinder where symmetry conditions (22) were considered. The outer parts correspond to the expansion fronts where the constant source of concentration $C_{l}^{e}$ is assumed. We observe a smooth solution that develops from low levels of concentration to high levels of concentration as expected, since the liquid penetration occurs from the outermost regions of the plot toward the axes.
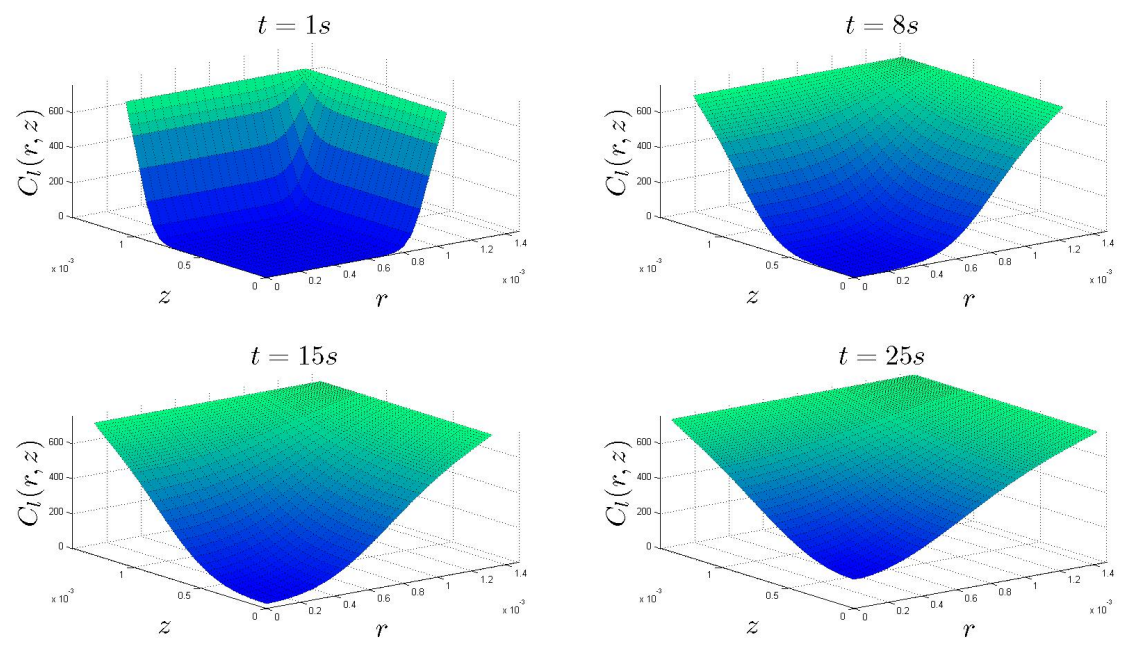

Figure 3: Concentration of solvent $C_{l}$, for different $t$

In Figures 4 we present plots of the concentration of dissolved drug at $t=1 \mathrm{~s}, t=8 \mathrm{~s}, t=15 \mathrm{~s}$ and $t=25 \mathrm{~s}$. As before, the axes $z$ and $r$ correspond to the inner part of the cylinder where symmetry conditions (22) were considered. The outermost part of the plots correspond to the expansion fronts where a perfect sink condition is assumed. We observe that regions where the concentration of the liquid solvent is high, correspond to regions where the concentration of dissolved drug is also high. 

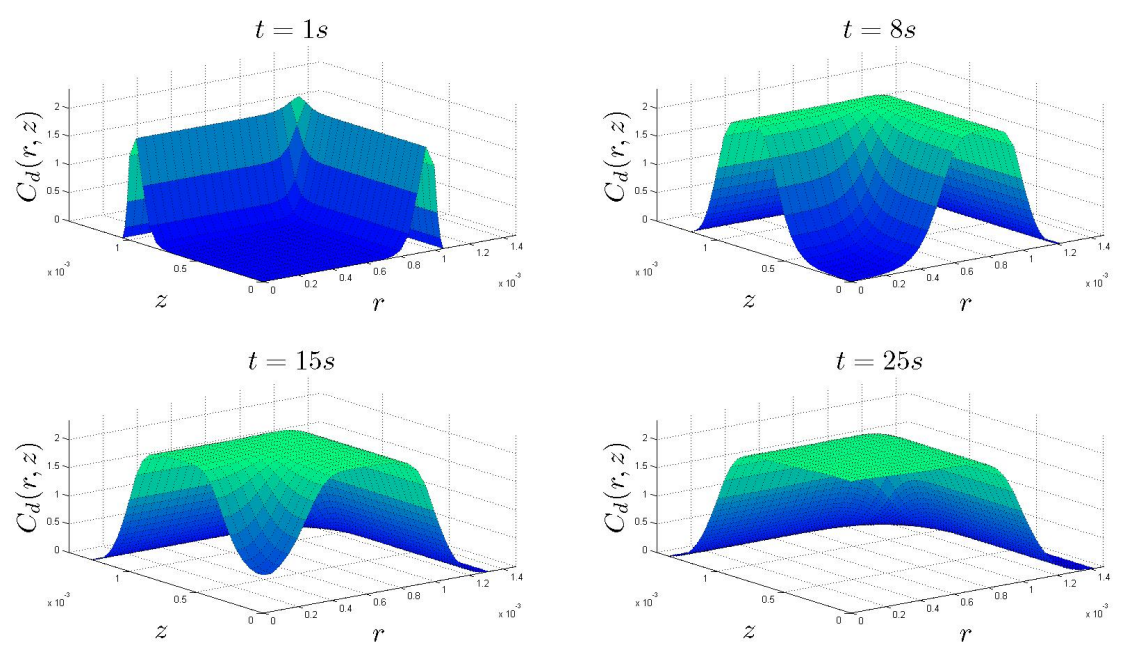

Figure 4: Concentration of dissolved drug $C_{d}$, for different $t$

In Figures 5 we show plots of the concentration of solid drug at $t=1 \mathrm{~s}, t=8 \mathrm{~s}, t=15 \mathrm{~s}$ and $t=25 \mathrm{~s}$. We observe that as the concentration of dissolved drug increases, the concentration of solid drug decreases smoothly towards the moving fronts. On the contrary to what is observed in the plots of dissolved drug, the regions of highest solid drug concentration correspond to regions of lowest liquid agent concentration.
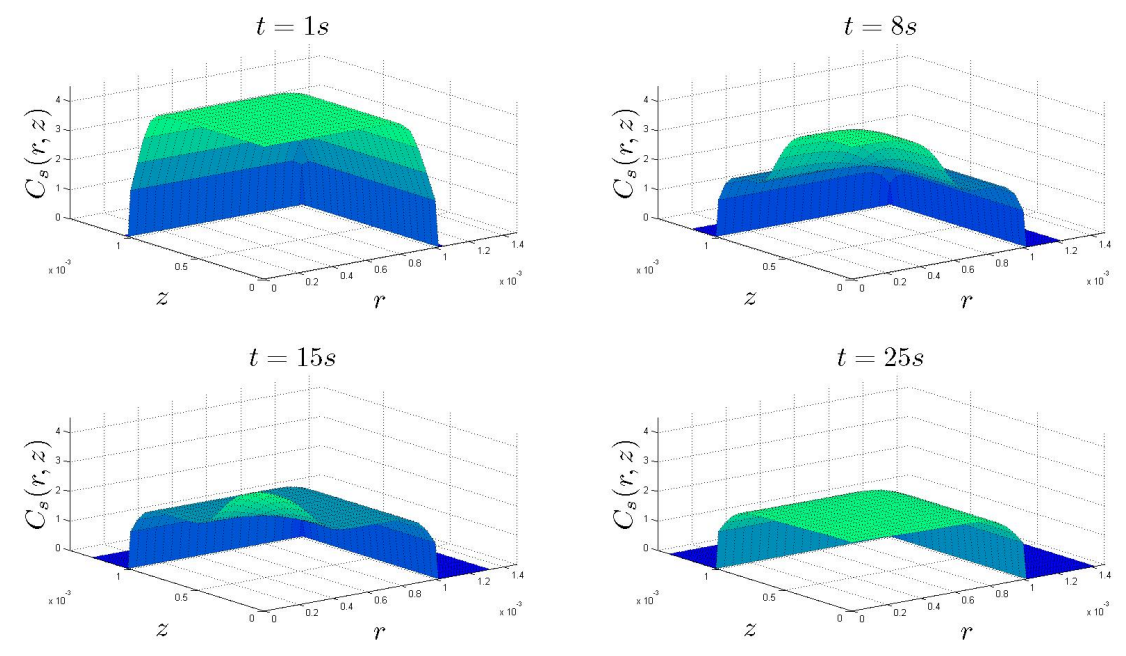

Figure 5: Concentration of undissolved $\operatorname{drug} C_{s}$, for different $t$

In Figures 6 and 7 we plotted the movement in time of the dimensionless swelling fronts in both axial and radial directions. We observe that in both cases, the initial uptake of the solvent produces an initial rapid growth of the swelling followed by an equilibrium state of the fronts.

According to Flory theory [8] there is a link between $E_{0}$ and $C_{l}^{e}$, more precisely, at equilibrium 


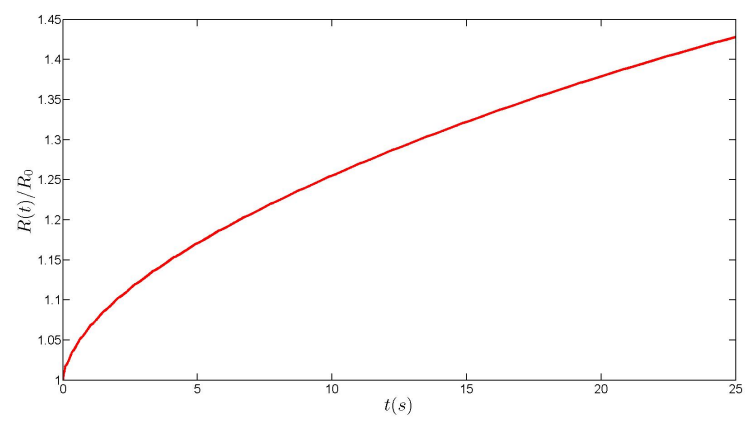

Figure 6: Swelling in the radial direction

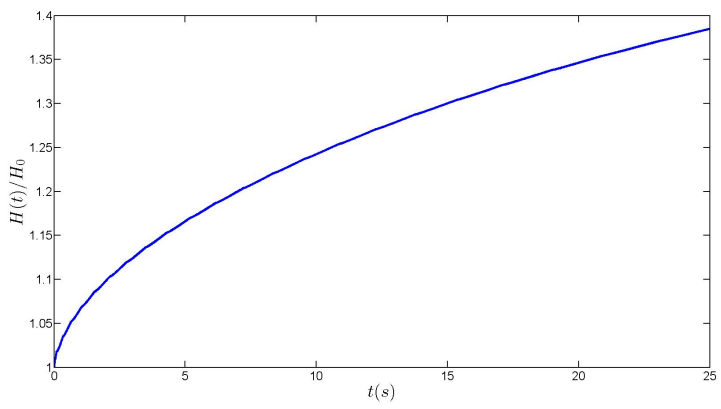

Figure 7: Swelling in the axial direction

we have

$$
\ln \left(1-\phi_{p}\right)+\phi_{p}+\chi \phi_{p}^{2}+\rho_{x} V_{1}\left(\phi_{p}^{\frac{1}{3}}-0.5 \phi_{p}\right)=0
$$

where $\phi_{p}$ represent the polymer volume fraction, $V_{1}$ the solvent molar volume, $\rho_{x}$ is the crosslink density and $\chi$ is the Flory interaction parameter. As $\rho_{x}$ can be computed with the formula

$$
\rho_{x}=\frac{E_{0}}{3 R_{g} T},
$$

where $R_{g}$ is the universal gas constant and $T$ the absolute temperature. Assuming that $\chi=0.6$, $T=298.15 \mathrm{~K}$ and $V_{1}=18.064 \times 10^{-6} \mathrm{~m}^{3} / \mathrm{mol}$, then once $E_{0}$ is fixed, $\phi_{p}$ can be calculated with (37) and the corresponding $C_{l}^{e}$ can be obtained from

$$
C_{l}=\rho_{l}\left(1-\phi_{p}\right)
$$

In Figures 8 and 9 we present the dimensionless swelling fronts as functions of the parameter $E_{0}$ and its corresponding $C_{l}^{e}$. In both cases we observe that the fronts are decreasing functions of $E_{0}$. We note that this behavior is physically sound, since an increase in $E_{0}$ corresponds to an increase in the resistance of the polymer to swelling.

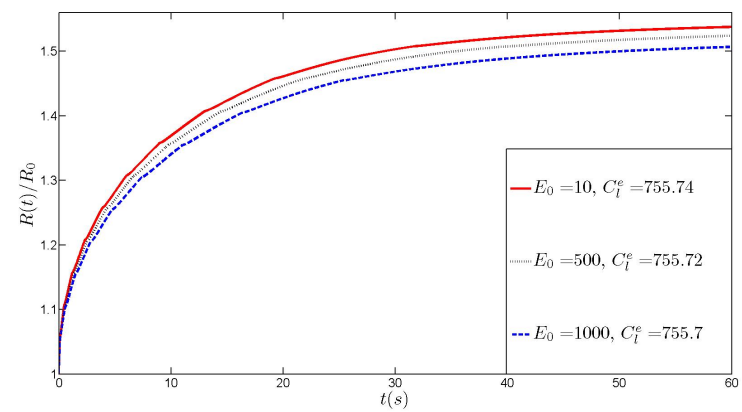

Figure 8: Radial swelling, $R(t) / R_{0}$ as a function of $E_{0}$

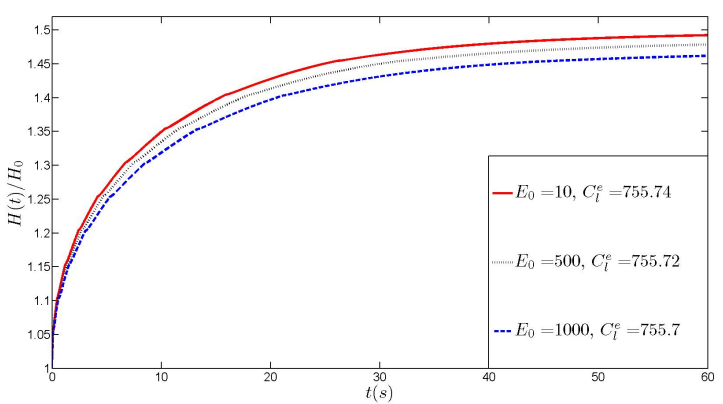

Figure 9: Axial swelling, $H(t) / H_{0}$ as a function of $E_{0}$

By $M_{d} / M_{d_{0}}(t)$, where $M_{d}$ is the total mass of drug released at time $t$ and $M_{d_{0}}$ is the initial mass loaded in the polymeric matrix, we represent the dimensionless total mass of drug released at 
time $t$ defined as

$$
M_{d} / M_{d_{0}}(t)=1-\frac{2}{R_{0}^{2} H_{0} C_{s}^{0}} \int_{0}^{H(t)} \int_{0}^{R(t)} r\left(C_{s}(r, z, t)+C_{d}(r, z, t)\right) d r d z
$$

and by $M_{s} / M_{\infty}(t)$ the mass of the liquid solvent inside of the matrix at time $t$, defined as

$$
M_{s} / M_{\infty}(t)=\frac{2}{R_{e q}^{2} H_{e q} C_{l}^{e}} \int_{0}^{H(t)} \int_{0}^{R(t)} r C_{l}(r, z, t) d r d z
$$

where $R_{e q}$ and $H_{e q}$ are the values of $R(t)$ and $H(t)$ at equilibrium, respectively.

In order the study the effects of swelling in drug release we plotted $M_{d} / M_{d_{0}}$ as a function of $E_{0}$ in Figures 10 and 11. In Figure 10 we assume that the polymer does not swell and in Figure 11 a moving boundary due to swelling is considered. When we say that no swelling is present we mean that $L(t)=L_{0}$ for all $t$. We observe in Figure 10 that $M_{d} / M_{d_{0}}$ is a decreasing function of $E_{0}$. Conversely in Figure 11 we observe that $M_{d} / M_{d_{0}}$ is an increasing function of $E_{0}$. As shown in Figures 8 and 9 , an $E_{0}$ decrease implies a swelling increase and, therefore, the dissolved drug has to travel a larger distance to the moving front. Consequently, less dissolved drug accumulates at the front where the perfect sink condition is assumed and the mass of drug released decreases as the swelling increases.

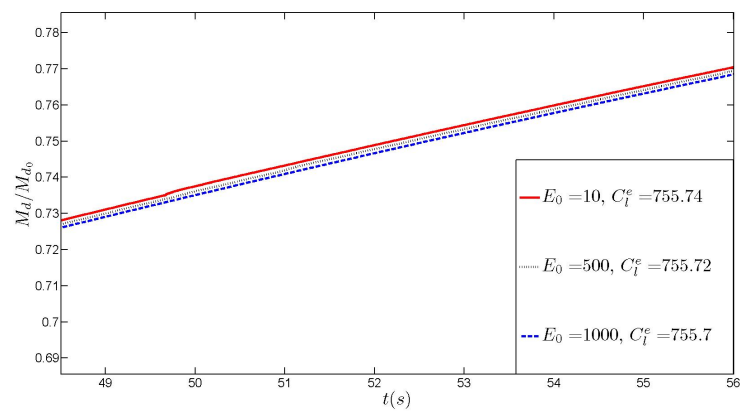

Figure 10: Mass of drug released, $M_{d} / M_{d_{0}}$ as a function of $E_{0}$ with $L(t)=L_{0}$

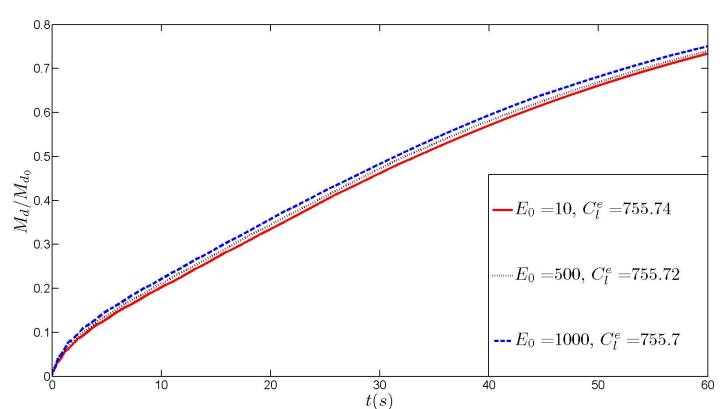

Figure 11: Mass of drug released, $M_{d} / M_{d_{0}}$ as a function of $E_{0}$

In Figures 12 and 13 we show plots of $M_{s} / M_{\infty}$ and $M_{d} / M_{d_{0}}$ respectively as a function of $\mu_{1}$. Figure 12 shows that $M_{s} / M_{\infty}$ is a decreasing function of $\mu_{1}$ and Figure 13 that $M_{d} / M_{d_{0}}$ is an increasing function of $\mu_{1}$. In Figure 12 we did a a 5 th degree polynomial fitting in order to avoid the jumps that appear as a consequence of the moving of the boundary.

Finally in Figure 14 we plotted $R(t) / H(t)$ for different initial values of $R_{0} / H_{0}$. As proved by Tanaka in [21], we observe that upon swelling $R(t) / H(t)$ is constant and approximately equal to $R_{0} / H_{0}$

\section{Conclusions}

In this paper a 3D mathematical model to describe the drug release from a cylindrical polymeric matrix is presented. We assume that a solvent diffuses into the matrix creating a stress driven 


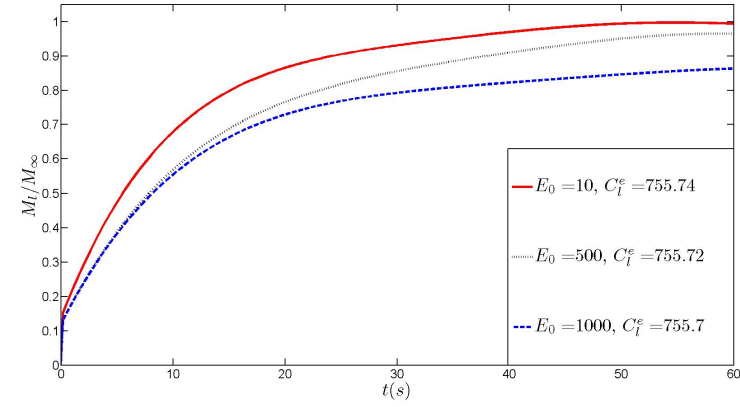

Figure 12: Mass of solvent, $M_{s} / M_{\infty}$ as a function of the viscosity $\mu_{1}$

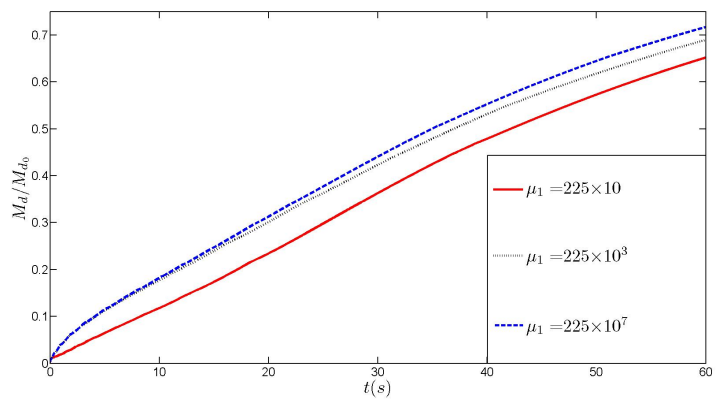

Figure 13: Mass of drug released, $M_{d} / M_{d_{0}}$ as a function of the viscosity $\mu_{1}$

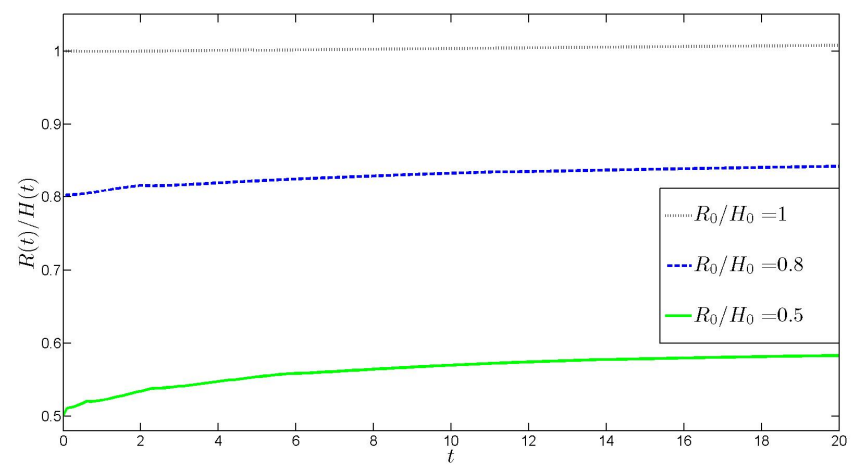

Figure 14: $R(t) / H(t)$ as a function of $R_{0} / H_{0}$

diffusion, thus a non-Fickian mass flux. To describe this phenomena, we consider a modified flux equation that is the sum of a Fickian flux $J_{F}$ and a non-Fickian flux $J_{N F}$. The viscoelastic behavior of the polymer is described by considering a mechanistic system of dampers and springs known as the generalized Maxwell-Wiechert model and by introducing concentration dependent functional relations for the strain and the viscoelastic diffusion coefficient. For the drug release, as we assume that the drug is present in two states dissolved and undissolved, the process is described by Fickian diffusion associated to solvent uptake coupled with non-linear dissolution. To describe the swelling of the polymeric cylinder we consider a volume conservation equation to track the movement of the fronts in both radial and axial directions. To solve the initial-boundary value problem associated to the system of equations we use an Implicit-Explicit (IMEX) method to obtain numerical solutions. We exhibit several plots to illustrate the behavior of the solutions and the influence that the parameters associated to the model have over the drug release.

The great advantage of the proposed model consists in the possibility of easily and directly incorporating experimental rheological information about polymer-solvent matrix system (knowledge of $E_{k}$ and $\mu_{k}$ ). Indeed, once the mechanical spectrum referring to a particular polymer-solvent matrix is experimentally determined by rheological tests (frequency sweep), the Maxwell-Wiechert model (also called generalized Maxwell model by rheologists) can be fitted to experimental data for the determination of $E_{k}$ and $\mu_{k}$ [14]. Of course, our model could be also implemented considering 
the dependence of $E_{k}$ and $\mu_{k}$ on local solvent concentration. Again, this information can be experimentally deduced by determining the mechanical spectrum referring to matrices characterized by an increasing polymer concentration. Finally it is worth mentioning that all the parameters of the model can be measured or estimated according to well-known theories.

Acknowledgements: this work was partially supported by the Centro de de Matemática da Universidade de Coimbra (CMUC), funded by the European Regional Development Fund through the program COMPETE and by the Portuguese Government through the FCT - Fundação para a Ciência e Tecnologia under the projects PEst-C/MAT/UI0324/2011, SFR/BD/33703/2009, UTAustin/MAT/0066/2008 and by Fondo PRIN 2010-2011 (20109PLMH2).

\section{References}

[1] H.F. Brinson, L.C. Brinson, Polymer Engineering Science and Viscoelasticity, An Introduction, Springer, 2008.

[2] G. Camera-Roda, G.C. Sarti, Mass transport with relaxation in polymers, AIChE Journal, 36: 851-860, 1990.

[3] D.S. Cohen, D.A. Edwards, A mathematical model for a dissolving polymer, AIChE Journal, 41: 2345-2355, 1995.

[4] D.S. Cohen, A. B. White Jr., Sharp fronts due to diffusion and viscoelastic relaxation in polymers, SIAM Journal Applied Mathematics, 51: 472-483, 1991.

[5] D.A. Edwards, Non-Fickian Diffusion in thin polymer films, Journal of Polymer Science, Part B: Polymer Physics Edition, 34: 981-997, 1996.

[6] J.A. Ferreira, E. Gudiño, P. de Oliveira, A second order approximation for quasilinear nonFickian diffusion models, Computational Methods in Applied Mathematics, 13(4): 471-493, 2013.

[7] J.A. Ferreira, M. Grassi, E. Gudino, P. de Oliveira, A new look to non-Fickian diffusion, preprint Universidade de Coimbra, 2013.

[8] P.J. Flory, Thermodynamics of high polymer solutions, J. Chem. Phys., 10:51-61, 1942.

[9] G. Fu, W.O. Soboyejo, Investigation of swellable poly (N-isopropylacrylamide) based hydrogels for drug delivery, Materials Science and Engineering C, 31: 1084-1090, 2011.

[10] H. Fujita, Diffusion in polymer-diluent systems, Fortschr Hochpolym Forsch, 3: 1-47, 1961.

[11] M. Grassi, G. Grassi, Mathematical modeling and controlled drug delivery: matrix systems, Current Drug Delivery, 2: 97-116, 2005.

[12] M. Grassi, G. Grassi, R. Lapasin, I. Colombo, Understanding Drug Release and Absorption Mechanisms: A Physical and Mathematical Approach, CRC Press, 2006.

[13] M. Grassi, S.H. Yuk, S.H. Cho, Modelling of solute transport a temperature-sensitive polymer, J. Membrane. Sci., 152: 241-249, 1999. 
[14] R. Lapasin, S. Pricl, Rheology of industrial polysaccharides: Theory and applications, London: Blackie Academic \& Professional, 1995.

[15] P.I. Lee, Diffusional release of a solute from a polymeric matrix-approximate analytical solutions. J. Membr. Sci., 7: 255-275, 1980.

[16] Q. Liu, D. De Kee, Modeling of diffusion through polymeric membranes, Rheologica Acta 44: 287-294, 2005.

[17] Q. Liu, X. Wang, D. De Kee, Mass transport through swelling membranes, International Journal of Engineering Science, 43: 1464-1470, 2005.

[18] D.R. Paul, S.K. McSpadden, Diddusional release of a solute from a polymer matrix, J. Membr. Sci., 1: 33-48, 1976.

[19] S. Shaw, J. R. Whiteman, Some partial differential Volterra equation problems arising in viscoelasticity, Proceeding of the Conference on Differential Equations and their Applications, Brno, August 25-29, 1997, ed. R. P. Agarwal, F. Neuman, J.Vosmanský, 183-200, 1998 .

[20] J. Siepmann, F. Siepmann, Mathematical modeling of drug delivery, International Journal of Pharmaceutics 364: 328-343, 2008.

[21] Y. Li, T. Tanaka, Effects of shear modulus of polymer gels, in Polymer Gels: fundamental and biomedical applications, DeRossi, D., Kajiwara, K., Osada, Y., Yamauchi, A. eds. Plenum Press, New York, 41-56, 1991.

[22] L.N. Thomas, A.H. Windle, A deformation model for case II diffusion, Polymer, 21: 613-619, 1980 .

[23] L.N. Thomas, A.H. Windle, Diffusion mechanics of the system PMMA-methano, Polymer, 22: 627-639, 1981.

[24] L.N. Thomas, A.H. Windle, A theory of case II diffusion, Polymer, 23: 529-542, 1982.

[25] D.E. Pelinovsky, W. Zhao, Multilevel computations of dispersed drug release, Numerical Methods for Partial Differential Equations, 29: 1391-1415, 2013.

[26] M.F. Wheeler, A priori $L^{2}$ error estimates for Galerkin approximation to parabolic partial differential equations, SIAM Journal on Numerical Analysis, 10: 723-759, 1973.

[27] X.Y. Wu, Y. Zhou, Studies of diffusional release of a dispersed solute from polymeric matrices by finite element method, J. Pharm. Sci, 88: 1050-1057, 1999. 